\title{
RENAL PHYSIOLOGY IN LOBAR PNEUMONIA ${ }^{1}$
}

\author{
By LEE E. FARR ANd T. J. ABERNETHY \\ (From the Hospital of the Rockefeller Institute for Medical Research, New York City)
}

(Received for publication December 23, 1936)

Previous studies on renal physiology during acute pneumococcus pneumonia have yielded rather discordant results. In general a hyperfunction has been reported, but this phenomenon has not always been observed. Lewis (1), McIntosh and Reimann (2) and Goldring (3) found an apparent increased functional capacity of the kidney during pneumonia. Rackemann, Longcope and Peters (4) and Frothingham (5) reported that there was neither impairment of function nor hyperfunction. On the other hand, Tileston and Comfort (6) Schwartz and McGill (7) and Bookman (8) reported definite evidence of renal impairment. Interest has been stimulated in the relation of the pneumococcus to renal pathology by the work of Blackman (9) and his coworkers on the association of this organism with the nephrotic syndrome. Rake (10) and Seegal (11) have reported on the incidence of nephritis in pneumococcus infection while Winkenwerder, McLeod and Baker (12) have tabulated the incidence of pneumonia preceding nephritis. In a survey of the literature by Rake, the pneumococcus was second to the streptococcus as a bacterial incitant of Bright's' disease, while Winkenwerder, McLeod and Baker found that pneumonia was the type of infection observed at the onset of 6.5 per cent of patients in their series with Bright's disease. Seegal, however, observed but seven cases of nephritis in 1004 cases of pneumonia. The reasons for the variations in these statistics are not clear.

The present series of observations was undertaken to determine the immediate and delayed effect of pneumococcus pneumonia on renal function and to gain some understanding of the changes in renal physiology accompanying the period of febrile illness and also convalescence.

The primary mode of approach to estimation of renal function was through the urea clearance test and estimations of protein excretion in the urine. These two procedures could be carried on without

\footnotetext{
1 Read by title at the meeting of the American Society for Clinical Investigation, in Atlantic City, May, 1936.
}

requiring too great cooperation of the patient and were independent of the treatment regime. Sediment counts were done on fresh morning specimens as an adjunct to the above tests. Other more elaborate tests were not done, as in many instances the patient was too ill to be disturbed.

\section{METHOD OF STUDY}

Twenty-eight patients admitted consecutively to the Rockefeller Institute for treatment of pneumonia were observed over varying periods of time, in some instances as long as 6 months. On admission a urea clearance test was done; the following day a sediment count was performed. Urea clearances were repeated every three days until crisis, when practicable, and were always done on the day of crisis. During the acute illness 12-hour clearances were done unless the patient was incontinent, when the periods were shortened to those over which urine collection was complete. Another urea clearance was done 5 days post-crisis and at the time of discharge. Following discharge, urea clearances were done on the patients in the Outpatient Clinic once a month until renal function had been normal for two months. Sediment counts were done on the first day after crisis and on the sixth day after crisis. Quantitative estimations of urinary protein by the method of Shevky and Stafford (13) were done daily during the febrile period and as long thereafter as protein was present. Blood pressure determinations were done daily, and retinal examinations were made on each patient during the acute illness as well as in convalescence. Urea clearances were done more frequently than noted above whenever the results were outside the usual normal range. The patients were studied without reference to the type of pneumococcus infections or method of treatment. The bacteriological findings and treatment of the disease in these patients are shown in Table I. None of the patients gave any history of preexisting renal disease. In none of the patients was any retinopathy noted nor was there any marked sclerosis 
TABLE I

\begin{tabular}{|c|c|c|c|c|c|c|}
\hline $\begin{array}{c}\text { Pneumococcus } \\
\text { type }\end{array}$ & Patient & Sex & Age & $\begin{array}{c}\text { Serum } \\
\text { ther- } \\
\text { apy }\end{array}$ & $\begin{array}{c}\text { Bac- } \\
\text { te- } \\
\text { re- } \\
\text { mia }\end{array}$ & Outcome \\
\hline I & $\begin{array}{l}\text { A. B. } \\
\text { M. A. } \\
\text { C. E. } \\
\text { P. T. } \\
\text { E. M. } \\
\text { H. Y. } \\
\text { R. L. }\end{array}$ & $\begin{array}{l}\mathrm{M} . \\
\mathbf{F} . \\
\mathbf{M} . \\
\mathbf{M} . \\
\mathrm{F} . \\
\mathrm{F} .\end{array}$ & $\begin{array}{c}\text { years } \\
17 \\
18 \\
15 \\
17 \\
29 \\
40 \\
42\end{array}$ & $\begin{array}{l}+ \\
+ \\
+ \\
+ \\
+ \\
+ \\
+\end{array}$ & $\begin{array}{l}\bar{t} \\
\bar{t} \\
\overline{-} \\
-\end{array}$ & $\begin{array}{l}\text { Recovered } \\
\text { Recovered } \\
\text { Recovered } \\
\text { Recovered } \\
\text { Recovered } \\
\text { Recovered } \\
\text { Recovered }\end{array}$ \\
\hline II & $\begin{array}{l}\text { A. } \mathrm{K} \text {. } \\
\text { S. M. }\end{array}$ & $\begin{array}{l}\mathbf{M} \\
\mathbf{M}\end{array}$ & $\begin{array}{l}30 \\
56\end{array}$ & + & $\overline{-}$ & $\begin{array}{l}\text { Recovered } \\
\text { Recovered }\end{array}$ \\
\hline III & $\begin{array}{l}\text { P. M. } \\
\text { M. S. } \\
\text { H. N. } \\
\text { M. C. } \\
\text { H. B. } \\
\text { N. H. } \\
\text { E. W. }\end{array}$ & $\begin{array}{l}\mathrm{M} \\
\mathbf{F} . \\
\mathbf{F} \\
\mathbf{M} \\
\mathbf{M} . \\
\mathbf{F} . \\
\mathbf{M}\end{array}$ & $\begin{array}{l}21 \\
48 \\
55 \\
56 \\
65 \\
29 \\
45 \\
\end{array}$ & $\begin{array}{l}\bar{z} \\
\bar{z} \\
\bar{z} \\
\bar{z}\end{array}$ & $\begin{array}{l}\bar{z} \\
\bar{z} \\
\bar{z}\end{array}$ & $\begin{array}{l}\text { Recovered } \\
\text { Recovered } \\
\text { Recovered } \\
\text { Recovered } \\
\text { Recovered } \\
\text { Recovered } \\
\text { Died }\end{array}$ \\
\hline V & $\begin{array}{l}\text { E. C. } \\
\text { O. S. }\end{array}$ & $\mathbf{F}$. & $\begin{array}{l}10 \\
39\end{array}$ & $\overline{-}$ & $\overline{-}$ & $\begin{array}{l}\text { Recovered } \\
\text { Recovered }\end{array}$ \\
\hline VII & $\begin{array}{l}\text { O. } \mathbf{K} . \\
\text { J. T. } \\
\dot{\mathrm{M}} . \dot{\mathrm{B}} .\end{array}$ & $\begin{array}{l}\mathbf{F} . \\
\mathbf{M} \\
\mathbf{M}\end{array}$ & $\begin{array}{l}22 \\
28 \\
20\end{array}$ & \pm & $\bar{z}$ & $\begin{array}{l}\text { Recovered } \\
\text { Recovered } \\
\text { Recovered }\end{array}$ \\
\hline VIII & $\begin{array}{l}\text { H. F. } \\
\text { A. I. }\end{array}$ & $\begin{array}{l}\mathbf{M} \\
\mathbf{M}\end{array}$ & $\begin{array}{l}35 \\
52\end{array}$ & $\overline{-}$ & $\overline{-}$ & $\begin{array}{l}\text { Recovered } \\
\text { Recovered }\end{array}$ \\
\hline IX & G. Z. & F. & 49 & - & - & Recovered \\
\hline Unclassifiable & $\begin{array}{l}\text { D. B. } \\
\text { S. M. } \\
\text { N. G. }\end{array}$ & $\begin{array}{l}\mathrm{F} . \\
\mathrm{F} . \\
\mathbf{M}\end{array}$ & $\begin{array}{l}10 \\
34 \\
36\end{array}$ & $\bar{z}$ & $\bar{z}$ & $\begin{array}{l}\text { Recovered } \\
\text { Recovered } \\
\text { Recovered }\end{array}$ \\
\hline $\begin{array}{l}\text { B. Friedländer } \\
\text { Type C }\end{array}$ & N. G. & M. & 50 & - & - & Died \\
\hline
\end{tabular}

of peripheral vessels. Blood cultures were done routinely on admission and were negative unless otherwise noted. The number of patients with each type of pneumococcus infection is summarized in Table I. The data on the two patients who died are shown in Table II-E.

\section{RESULTS}

Urea clearance. Renal function during the febrile period before crisis as revealed by the urea clearance test varied inversely as the age of the patient. This is demonstrated in Figure 1 and Tables II-A to II-E. During the febrile period the average urea clearance for eleven patients in the age group 10 to 29 years was elevated to 147 per cent of normal. The average for four pa- tients 30 to 39 years was 111.8 per cent of normal; of five patients 40 to 49 years, 97.7 per cent normal ; and of five patients 50 to 65 years, 82.6 per cent of normal. Thus it would appear that renal function during the pre-critical period of the disease may be elevated, may be unchanged, or may be slightly impaired, and that the level is in general a function of the age of the patient. The clearances done immediately after crisis and while the patient was still in the hospital do not show a rapid return to normal. On the contrary, the clearance in the younger group remained elevated for approximately a month after the onset of the disease. At no time during the acute illness did any of these younger patients have a urea clearance below 110 per cent of normal. In fact, all of the clearance figures at this time were 130 per cent or above, except in three instances shown in Figure 1. In each of these cases the clearance was done before fluid equilibrium had been established, and it was felt that the lower values so obtained $(110.5,113$ and 113 per cent respectively) could be explained in part on this basis. Furthermore, during the first 20 days of the disease a majority of the urea clearance figures were elevated to 130 per cent or above except in 3 additional instances (Cases E. M., C. E., and N. H.11th, 14th, and 15th days of disease). Fluid equilibrium had been established in these patients, however, and the reason for the lack of hyperfunction was not apparent.

In the age group 30 to 40 years, there was only moderate hyperfunction during the first ten days of the disease, and again in no instance was hypofunction noted. The number of patients in this group, however, is too small to warrant any conclusions.

In the age group 40 to 50 years, there was a definite absence of any increase of the urea clearance during the acute and convalescent periods of the illness. The clearance returned to an average value for this group in about three months. It is interesting to note that in this group of patients there is an apparent hyperfunction late in convalescence, occurring during the third month after onset, and that it is only after ninety days from onset that normal clearances are again observed. It was in this group that the lowest clearance was noted (first clearance 10.3 per cent on Case 
H. Y.). The reason for this low value was not apparent.

In the oldest group there is no definite shift in the clearance below normal minimum, but the values during the acute illness are in general somewhat below normal average. No secondary variations in clearance values were noted in this group.

Blood urea nitrogen. The blood urea nitrogen in all of the patients at the time of admission was that the patient was dehydrated. With restoration of fluids normal function followed, extra renal factors playing a predominant rôle.

Proteinuria. Contrary to expectation, the amount of protein lost in the urine by these patients during the febrile period was negligible. The majority of patients excreted less than 0.1 gram of protein per day throughout their entire hospital stay. In only one instance (E. C.) did

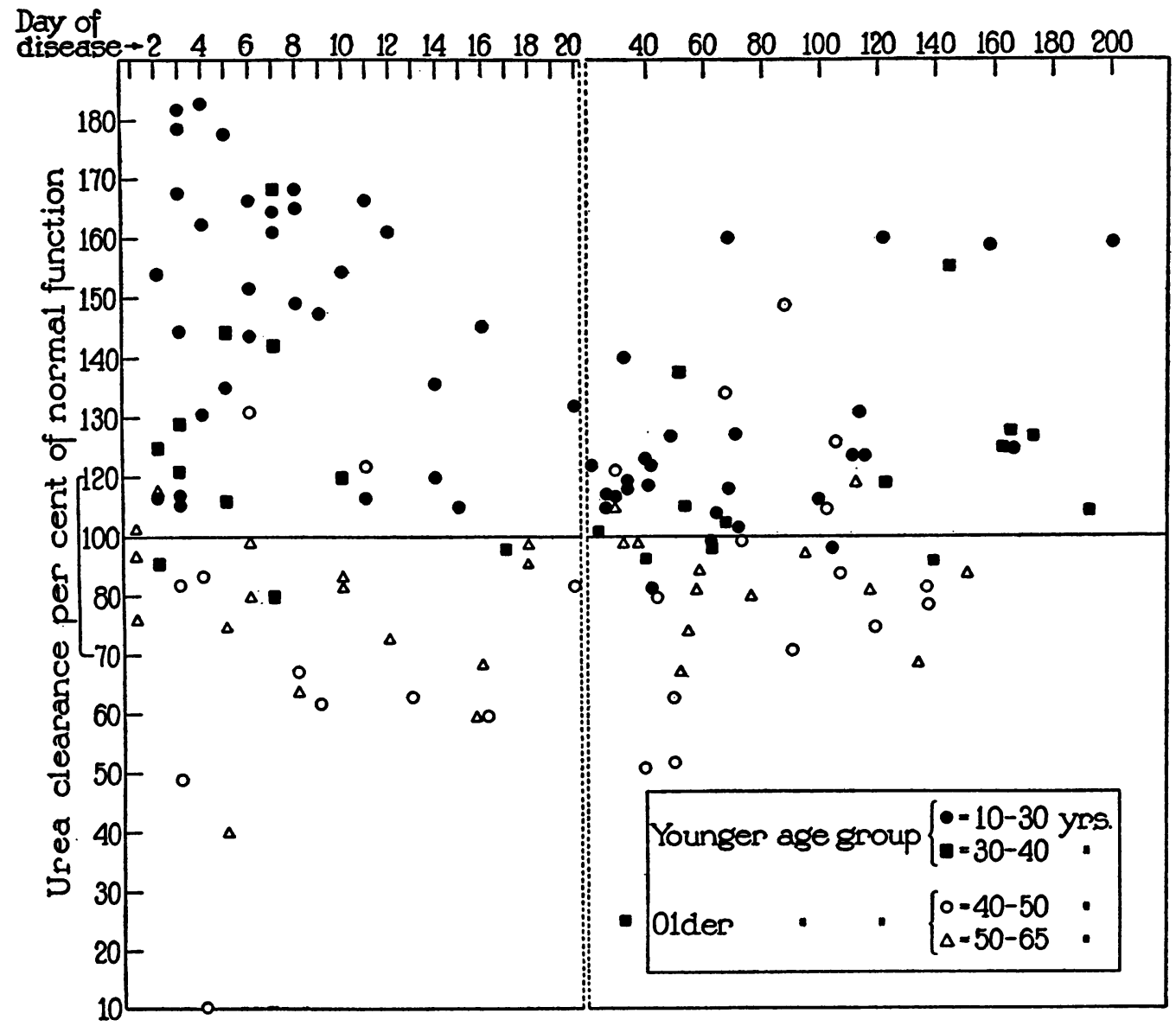

Fig. 1. Scatter Chart Showing Relation of Urea Clearance Changes to Day of Disease.

within normal limits except for two patients (H. Y. and S. M.). The clearances, however, on admission, differed widely. In both exceptions noted above the blood urea nitrogen had dropped to normal five days later. In the patient $\mathrm{H}$. $\mathrm{Y}$. (Table II-C) there is a discrepancy between the clearance and the expected and observed blood urea nitrogen. Why this blood urea nitrogen should be lower than expected with a function of 10 per cent is not clear, unless it mirrors the fact the protein excretion become significant, and in this patient the excretion never exceeded 3.0 grams per day and lasted for only four days. The early administration of adequate amounts of fluid to these patients seemed to depress protein excretion.

Sediment counts. In no single instance was the number of red blood cells excreted in 12 hours greater than the normal values defined by Addis. The difficulties of doing red blood cell counts on 
TABLE II

Summary of laboratory examinations on patients while under observation

\begin{tabular}{|c|c|c|c|c|c|c|c|c|c|c|c|c|c|c|}
\hline \multirow{4}{*}{ Patient } & \multirow{4}{*}{ Age } & \multicolumn{4}{|c|}{ Urea clearance } & \multicolumn{9}{|c|}{ Sediment and protein in 12-hrs. urine (no fluid restriction) } \\
\hline & & \multirow{3}{*}{$\begin{array}{c}\text { Day } \\
\text { of } \\
\text { dis- } \\
\text { ease }\end{array}$} & \multirow{3}{*}{$\begin{array}{c}\text { Day } \\
\text { before } \\
\text { or } \\
\text { after } \\
\text { crisis }\end{array}$} & \multirow{3}{*}{$\begin{array}{c}\text { Blood } \\
\text { urea } \\
\text { nitrogen }\end{array}$} & \multirow{3}{*}{$\begin{array}{c}\text { Urea } \\
\text { clear- } \\
\text { ance, } \\
\text { average } \\
\text { normal }\end{array}$} & \multirow{3}{*}{$\begin{array}{c}\text { Day } \\
\text { of } \\
\text { dis- } \\
\text { ease }\end{array}$} & \multirow{3}{*}{$\begin{array}{c}\text { Vol- } \\
\text { ume } \\
\text { in } \\
12 \\
\text { hours }\end{array}$} & \multicolumn{5}{|c|}{ Sediment } & \multirow{3}{*}{\multicolumn{2}{|c|}{ Proteinuria }} \\
\hline & & & & & & & & \multirow{2}{*}{$\mathrm{pH}$} & R. B. C. & \multicolumn{3}{|c|}{ Casts } & & \\
\hline & & & & & & & & & $\begin{array}{c}\text { Total } \\
\text { number } \\
\text { in 12 } \\
\text { hours }\end{array}$ & $\begin{array}{c}\begin{array}{c}\text { Total } \\
\text { number } \\
\text { in } 12 \\
\text { hours }\end{array} \\
\text { heurs }\end{array}$ & $\begin{array}{l}\text { Hya- } \\
\text { line }\end{array}$ & $\begin{array}{l}\text { Gran- } \\
\text { ular }\end{array}$ & & \\
\hline & years & & & $\underset{\text { per cent }}{\text { mgm. }}$ & per cent & & cc. & & & & $\begin{array}{l}\text { per } \\
\text { cent }\end{array}$ & $\begin{array}{l}\text { per } \\
\text { cent }\end{array}$ & $\underset{\text { per liter }}{\text { grams }}$ & $\begin{array}{l}\text { grams per } \\
18 \text { hours }\end{array}$ \\
\hline
\end{tabular}

A. PATIENTS 10 TO 29 YEARS

\begin{tabular}{|c|c|c|c|c|c|c|c|c|c|c|c|c|c|c|}
\hline E. C. $\%$ & 10 & $\begin{array}{r}4 \\
8 \\
21 \\
34 \\
\end{array}$ & $\begin{array}{l}-3 \\
+1 \\
+14 \\
+27\end{array}$ & $\begin{array}{r}8.17 \\
12.72 \\
8.59 \\
6.54\end{array}$ & $\begin{array}{l}162.1 \\
165.0 \\
122.7 \\
109.5\end{array}$ & $\begin{array}{l}4 \\
5 \\
6 \\
7\end{array}$ & $\begin{array}{l}858 \\
305 \\
493 \\
514\end{array}$ & & $\begin{array}{c}\text { No } \\
\text { counts } \\
\text { done }\end{array}$ & $\begin{array}{c}\text { No } \\
\text { counts } \\
\text { done }\end{array}$ & $\begin{array}{l}+ \\
\pm \\
\pm \\
-\end{array}$ & $\begin{array}{l} \pm \\
- \\
-\end{array}$ & $\begin{array}{c}0.7 \\
1.58 \\
1.8 \\
<0.07 \\
\end{array}$ & $\begin{array}{l}0.5 \\
0.8 \\
0.9\end{array}$ \\
\hline D. B. $\%$ & 10 & $\begin{array}{r}3 \\
5 \\
35 \\
172\end{array}$ & $\begin{array}{c}-2 \\
0 \\
+30 \\
+167\end{array}$ & $\begin{array}{r}8.82 \\
10.70 \\
7.15 \\
6.57\end{array}$ & $\begin{array}{l}110.5 \\
135.2 \\
113.6 \\
125.2\end{array}$ & $\begin{array}{r}5 \\
10\end{array}$ & $\begin{array}{l}850 \\
390\end{array}$ & $\begin{array}{l}6.0 \\
6.0\end{array}$ & $\begin{array}{l}0 \\
0\end{array}$ & $\begin{array}{l}\mathbf{0} \\
\mathbf{0}\end{array}$ & & & $\begin{array}{r}0.10 \\
<0.07 \\
\end{array}$ & 0.08 \\
\hline C. E. ఠ & 15 & $\begin{array}{r}3 \\
7 \\
14 \\
27 \\
34 \\
91\end{array}$ & $\begin{array}{c}0 \\
+4 \\
+11 \\
+24 \\
+31 \\
+88\end{array}$ & $\begin{array}{r}17.75 \\
11.39 \\
10.65 \\
7.90 \\
10.45 \\
7.50\end{array}$ & $\begin{array}{l}142.2 \\
164.1 \\
119.8 \\
114.3 \\
117.6\end{array}$ & $\begin{array}{r}4 \\
8 \\
14\end{array}$ & $\begin{array}{l}324 \\
500 \\
330\end{array}$ & $\begin{array}{l}8.0 \\
5.0 \\
4+\end{array}$ & $\begin{array}{c}24,000 \\
0 \\
0\end{array}$ & $\begin{array}{c}12,000 \\
0 \\
0\end{array}$ & 100 & & $\begin{array}{l}<0.07 \\
<0.07 \\
<0.07\end{array}$ & \\
\hline A. B. $\sigma^{\top}$ & 17 & $\begin{array}{r}2 \\
20 \\
122 \\
159 \\
201\end{array}$ & $\begin{array}{c}0 \\
+18 \\
+120 \\
+157 \\
+199\end{array}$ & $\begin{array}{l}12.26 \\
10.80 \\
11.29 \\
10.20 \\
11.65\end{array}$ & $\begin{array}{l}154.5 \\
132.0 \\
160.9 \\
158.5 \\
159.3\end{array}$ & $\begin{array}{r}4 \\
21\end{array}$ & $\begin{array}{l}412 \\
575\end{array}$ & \begin{tabular}{|l|}
6.0 \\
5.0
\end{tabular} & $\begin{array}{c}65,000 \\
0\end{array}$ & $\begin{array}{l}20,000 \\
18,000\end{array}$ & $\begin{array}{l}100 \\
100\end{array}$ & & $\begin{array}{r}1.15 \\
<0.07 \\
\end{array}$ & 0.48 \\
\hline P. T. $\sigma^{7}$ & 17 & $\begin{array}{r}4 \\
9 \\
33 \\
65 \\
100\end{array}$ & $\begin{array}{l}-1 \\
+4 \\
+28 \\
+60 \\
+95\end{array}$ & $\begin{array}{r}17.23 \\
13.59 \\
9.25 \\
10.80 \\
13.65\end{array}$ & $\begin{array}{l}130.6 \\
147.7 \\
140.4 \\
107.5 \\
112.4\end{array}$ & $\begin{array}{r}5 \\
11\end{array}$ & $\begin{array}{r}1,000 \\
895\end{array}$ & $\begin{array}{l}4+ \\
4+\end{array}$ & $\begin{array}{l}0 \\
0\end{array}$ & $\begin{array}{c}100,000 \\
0\end{array}$ & 100 & & $<0.07$ & \\
\hline M. A. ? & 18 & $\begin{array}{r}3 \\
7 \\
41 \\
69 \\
113\end{array}$ & $\begin{array}{c}0 \\
+4 \\
+38 \\
+66 \\
+110 \\
\end{array}$ & $\begin{array}{r}7.12 \\
7.07 \\
7.87 \\
8.47 \\
11.67 \\
\end{array}$ & $\begin{array}{l}167.4 \\
160.9 \\
117.2 \\
116.8 \\
131.0 \\
\end{array}$ & $\begin{array}{l}3 \\
8\end{array}$ & $\begin{array}{r}540 \\
1,090\end{array}$ & $\begin{array}{l}6.0 \\
7.0\end{array}$ & $\begin{array}{l}\mathbf{0} \\
\mathbf{0}\end{array}$ & $\begin{array}{c}16,000 \\
0\end{array}$ & 10 & 90 & 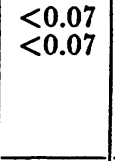 & \\
\hline M. B. ब' & 20 & $\begin{array}{r}6 \\
8 \\
14 \\
40 \\
69 \\
116\end{array}$ & $\begin{array}{l}-1 \\
+1 \\
+7 \\
+33 \\
+62 \\
+109\end{array}$ & $\begin{array}{r}19.39 \\
15.78 \\
12.24 \\
8.02 \\
9.49 \\
17.57\end{array}$ & $\begin{array}{l}143.7 \\
168.3 \\
135.5 \\
123.4 \\
160.6 \\
124.4\end{array}$ & $\begin{array}{r}3 \\
10 \\
14\end{array}$ & $\begin{array}{l}415 \\
165 \\
660\end{array}$ & $\begin{array}{l}4+ \\
6.0 \\
4+\end{array}$ & $\begin{array}{c}120,000 \\
0 \\
0\end{array}$ & $\begin{array}{c}12,000 \\
0 \\
0\end{array}$ & 100 & & $\begin{array}{l}<0.07 \\
<0.07 \\
<0.07\end{array}$ & \\
\hline P. M. $\sigma^{7}$ & 21 & $\begin{array}{r}3 \\
8 \\
49 \\
71\end{array}$ & $\begin{array}{r}0 \\
+5 \\
+46 \\
+68\end{array}$ & $\begin{array}{l}17.76 \\
12.22 \\
12.34 \\
12.95\end{array}$ & $\begin{array}{l}178.5 \\
149.0 \\
127.3 \\
127.2\end{array}$ & $\begin{array}{l}4 \\
9\end{array}$ & $\begin{array}{l}740 \\
420\end{array}$ & \begin{tabular}{|l|}
7.0 \\
7.0
\end{tabular} & $\begin{array}{r}8,000 \\
40,000\end{array}$ & $\stackrel{0}{25,000}$ & 100 & & $\begin{array}{r}0.36 \\
<0.07\end{array}$ & 0.28 \\
\hline O. K. $\%$ & 22 & \begin{tabular}{|r|}
3 \\
6 \\
12 \\
63 \\
112
\end{tabular} & $\begin{array}{l}-4 \\
-1 \\
+5 \\
+56 \\
+105\end{array}$ & $\begin{array}{l}5.21 \\
5.44 \\
7.72 \\
5.46 \\
5.16\end{array}$ & $\begin{array}{r}181.3 \\
166.1 \\
161.2 \\
98.0 \\
123.9\end{array}$ & $\begin{array}{r}4 \\
13\end{array}$ & $\begin{array}{r}2,550 \\
945\end{array}$ & $\begin{array}{l}6.0 \\
6.0\end{array}$ & $\begin{array}{c}0 \\
550,000\end{array}$ & $\begin{array}{c}0 \\
200,000\end{array}$ & 100 & & $\begin{array}{l}<0.07 \\
<0.07\end{array}$ & \\
\hline
\end{tabular}


TABLE II-Continued

\begin{tabular}{|c|c|c|c|c|c|c|c|c|c|c|c|c|c|c|}
\hline \multirow{4}{*}{ Patient } & \multirow{4}{*}{ Age } & \multicolumn{4}{|c|}{ Urea clearance } & \multicolumn{9}{|c|}{ Sediment and protein in 12-hrs. urine (no fluid restriction) } \\
\hline & & \multirow{3}{*}{$\begin{array}{c}\text { Day } \\
\text { of } \\
\text { dis- } \\
\text { ease }\end{array}$} & \multirow{3}{*}{$\begin{array}{c}\text { Day } \\
\text { before } \\
\text { or } \\
\text { after } \\
\text { crisis }\end{array}$} & \multirow{3}{*}{$\begin{array}{c}\text { Blood } \\
\text { urea } \\
\text { nitrogen }\end{array}$} & \multirow{3}{*}{$\begin{array}{c}\text { Urea } \\
\text { clear- } \\
\text { ance, } \\
\text { average } \\
\text { normal }\end{array}$} & \multirow{3}{*}{$\begin{array}{l}\text { Day } \\
\text { of } \\
\text { dis- } \\
\text { ease }\end{array}$} & \multirow{3}{*}{$\begin{array}{c}\text { Vol- } \\
\text { ume } \\
\text { in } \\
12 \\
\text { hours }\end{array}$} & \multicolumn{5}{|c|}{ Sediment } & & \\
\hline & & & & & & & & \multirow[b]{2}{*}{$\mathbf{p H}$} & R. B. C. & \multicolumn{3}{|c|}{ Casts } & \multirow{2}{*}{\multicolumn{2}{|c|}{ Proteinuria }} \\
\hline & & & & & & & & & $\begin{array}{c}\text { Total } \\
\text { number } \\
\text { in } 12 \\
\text { hours }\end{array}$ & $\begin{array}{c}\text { Total } \\
\text { number } \\
\text { in } 12 \\
\text { hours }\end{array}$ & $\begin{array}{l}\text { Hya- } \\
\text { line }\end{array}$ & $\underset{\text { ular }}{\text { Gran- }}$ & & \\
\hline & years & & & per cent & per cent & & cc. & & & & $\begin{array}{l}\text { per } \\
\text { cent }\end{array}$ & $\begin{array}{c}\text { per } \\
\text { cent }\end{array}$ & $\underset{\text { per liter }}{\text { grams }}$ & $\begin{array}{l}\text { grams per } \\
12 \text { hours }\end{array}$ \\
\hline
\end{tabular}

A. PATIENTS 10 TO 29 YEARS-(Continued)

\begin{tabular}{|c|c|c|c|c|c|c|c|c|c|c|c|c|c|c|}
\hline J. T. ه & 28 & $\begin{array}{r}2 \\
6 \\
11 \\
16 \\
43 \\
105\end{array}$ & $\begin{array}{l}-6 \\
-2 \\
+3 \\
+8 \\
+35 \\
+97\end{array}$ & $\begin{array}{r}15.97 \\
15.90 \\
10.88 \\
10.88 \\
9.33 \\
10.47\end{array}$ & $\begin{array}{r}113.5 \\
151.5 \\
166.4 \\
145.0 \\
82.0 \\
96.7\end{array}$ & $\begin{array}{r}3 \\
8 \\
12 \\
17\end{array}$ & $\begin{array}{l}370 \\
950 \\
442 \\
690\end{array}$ & $\begin{array}{l}6.0 \\
7.0 \\
5.0\end{array}$ & $\begin{array}{c}0 \\
0 \\
45,000 \\
20,000\end{array}$ & $\begin{array}{r}20,000 \\
70,000 \\
8,000 \\
50,000\end{array}$ & $\begin{array}{r}100 \\
100 \\
50 \\
100\end{array}$ & 50 & $\begin{array}{r}0.07 \\
<0.07 \\
<0.07 \\
<0.07\end{array}$ & 0.02 \\
\hline E. M. $\%$ & 29 & $\begin{array}{r}3 \\
5 \\
11 \\
34\end{array}$ & $\begin{array}{r}-2 \\
0 \\
+6 \\
+29\end{array}$ & $\begin{array}{r}7.15 \\
9.44 \\
12.20 \\
7.01\end{array}$ & $\begin{array}{l}113.6 \\
177.6 \\
113.6 \\
118.4\end{array}$ & $\begin{array}{r}4 \\
6 \\
13\end{array}$ & $\begin{array}{r}950 \\
430 \\
1,190\end{array}$ & $\begin{array}{l}7.0 \\
7.0 \\
7.0\end{array}$ & $\begin{array}{l}\mathbf{0} \\
\mathbf{0} \\
\mathbf{0}\end{array}$ & $\begin{array}{l}30,000 \\
45,000 \\
30,000\end{array}$ & $\begin{array}{r}100 \\
66 \\
100\end{array}$ & 33 & $\begin{array}{l}<0.07 \\
<0.07 \\
<0.07\end{array}$ & \\
\hline N. H. $?$ & 29 & $\begin{array}{r}4 \\
10 \\
15 \\
42 \\
72\end{array}$ & $\begin{array}{l}-4 \\
+2 \\
+7 \\
+34 \\
+64\end{array}$ & $\begin{array}{r}8.40 \\
5.66 \\
11.95 \\
7.46 \\
7.36\end{array}$ & $\begin{array}{l}182.4 \\
154.2 \\
110.3 \\
122.0 \\
103.0\end{array}$ & $\begin{array}{r}5 \\
10 \\
15\end{array}$ & $\begin{array}{l}220 \\
550 \\
520\end{array}$ & $\begin{array}{l}4+ \\
8.0 \\
7.0\end{array}$ & $\begin{array}{c}250,000 \\
0 \\
0\end{array}$ & $\begin{array}{c}185,000 \\
0 \\
30,000\end{array}$ & $\begin{array}{r}60 \\
100\end{array}$ & 40 & $\begin{array}{l}<0.07 \\
<0.07 \\
<0.07\end{array}$ & \\
\hline
\end{tabular}

B. PATIENTS 30 TO 39 YEARS

\begin{tabular}{|c|c|c|c|c|c|c|c|c|c|c|c|c|c|c|}
\hline A. K. $\sigma^{7}$ & 30 & $\begin{array}{r}3 \\
7 \\
54\end{array}$ & $\begin{array}{r}0 \\
+4 \\
+51\end{array}$ & $\begin{array}{l}19.46 \\
16.00 \\
16.32\end{array}$ & $\begin{array}{l}120.6 \\
142.0 \\
109.9\end{array}$ & $\begin{array}{l}3 \\
7\end{array}$ & $\begin{array}{l}380 \\
359\end{array}$ & $\begin{array}{l}5.0 \\
7.0\end{array}$ & $\begin{array}{c}9,000 \\
0\end{array}$ & $\begin{array}{r}18,000 \\
6,000\end{array}$ & $\begin{array}{l}100 \\
100\end{array}$ & & $\begin{array}{r}0.10 \\
<0.07\end{array}$ & 0.04 \\
\hline H. F. ه & 35 & $\begin{array}{r}2 \\
5 \\
10 \\
52 \\
121 \\
140 \\
174\end{array}$ & $\begin{array}{l}-2 \\
+1 \\
+6 \\
+48 \\
+117 \\
+136 \\
+170\end{array}$ & $\begin{array}{r}18.96 \\
11.82 \\
11.24 \\
8.12 \\
6.66 \\
7.06 \\
9.21\end{array}$ & $\begin{array}{r}91.2 \\
144.1 \\
120.0 \\
138.1 \\
145.0 \\
91.9 \\
126.9\end{array}$ & $\begin{array}{r}3 \\
7 \\
10\end{array}$ & $\begin{array}{l}540 \\
910 \\
920\end{array}$ & $\begin{array}{l}7.0 \\
5.0 \\
6.0\end{array}$ & $\begin{array}{l}0 \\
0 \\
0\end{array}$ & $\begin{array}{l}20,000 \\
55,000 \\
60,000\end{array}$ & & & $\begin{array}{r}0.28 \\
<0.07 \\
<0.07\end{array}$ & 0.14 \\
\hline N. G. ๙ & 36 & $\begin{array}{r}5 \\
17 \\
63 \\
123\end{array}$ & $\begin{array}{l}-3 \\
+9 \\
+54 \\
+114\end{array}$ & $\begin{array}{l}19.38 \\
11.69 \\
11.49 \\
13.46\end{array}$ & $\begin{array}{r}111.3 \\
95.8 \\
98.1 \\
119.0\end{array}$ & $\begin{array}{r}5 \\
16\end{array}$ & $\begin{array}{l}407 \\
610\end{array}$ & $\begin{array}{l}5.0 \\
7.0\end{array}$ & $\begin{array}{c}16,000 \\
0\end{array}$ & $\begin{array}{l}220,000 \\
180,000\end{array}$ & $\begin{array}{r}60 \\
100\end{array}$ & 40 & $\begin{array}{r}0.50 \\
<0.07\end{array}$ & 0.20 \\
\hline O. S. ब & 39 & $\begin{array}{r}2 \\
7 \\
24 \\
68 \\
165\end{array}$ & $\begin{array}{c}-5 \\
0 \\
+17 \\
+61 \\
+158\end{array}$ & $\begin{array}{l}15.17 \\
33.13 \\
11.43 \\
10.61 \\
15.97\end{array}$ & $\begin{array}{r}125.0 \\
79.0 \\
101.6 \\
104.6 \\
128.3\end{array}$ & $\begin{array}{r}9 \\
28\end{array}$ & $\begin{array}{l}830 \\
700\end{array}$ & $\begin{array}{l}4+ \\
6.0\end{array}$ & $\begin{array}{l}\mathbf{0} \\
\mathbf{0}\end{array}$ & $\begin{array}{r}370,000 \\
20,000\end{array}$ & $\begin{array}{r}60 \\
100\end{array}$ & 40 & $\begin{array}{l}<0.07 \\
<0.07\end{array}$ & \\
\hline S. M. $\%$ & 34 & $\begin{array}{r}3 \\
7 \\
33 \\
40 \\
145 \\
164 \\
194\end{array}$ & $\begin{array}{l}-3 \\
+1 \\
+27 \\
+34 \\
+139 \\
+158 \\
+188\end{array}$ & $\begin{array}{r}8.71 \\
6.24 \\
26.43 \\
6.60 \\
7.59 \\
9.50 \\
11.36\end{array}$ & $\begin{array}{r}129.5 \\
168.0 \\
25.1 \\
92.8 \\
155.3 \\
125.0 \\
108.9\end{array}$ & $\begin{array}{r}3 \\
8 \\
34\end{array}$ & $\begin{array}{l}215 \\
550 \\
360\end{array}$ & $\begin{array}{l}4+ \\
6.0\end{array}$ & $\begin{array}{c}30,000 \\
50,000 \\
0\end{array}$ & $\begin{array}{c}22,000 \\
85,000 \\
0\end{array}$ & $\begin{array}{l}100 \\
100\end{array}$ & & $\begin{aligned} & 2.6 \\
< & 0.07 \\
< & 0.07\end{aligned}$ & 0.46 \\
\hline
\end{tabular}


TABLE I-Continued

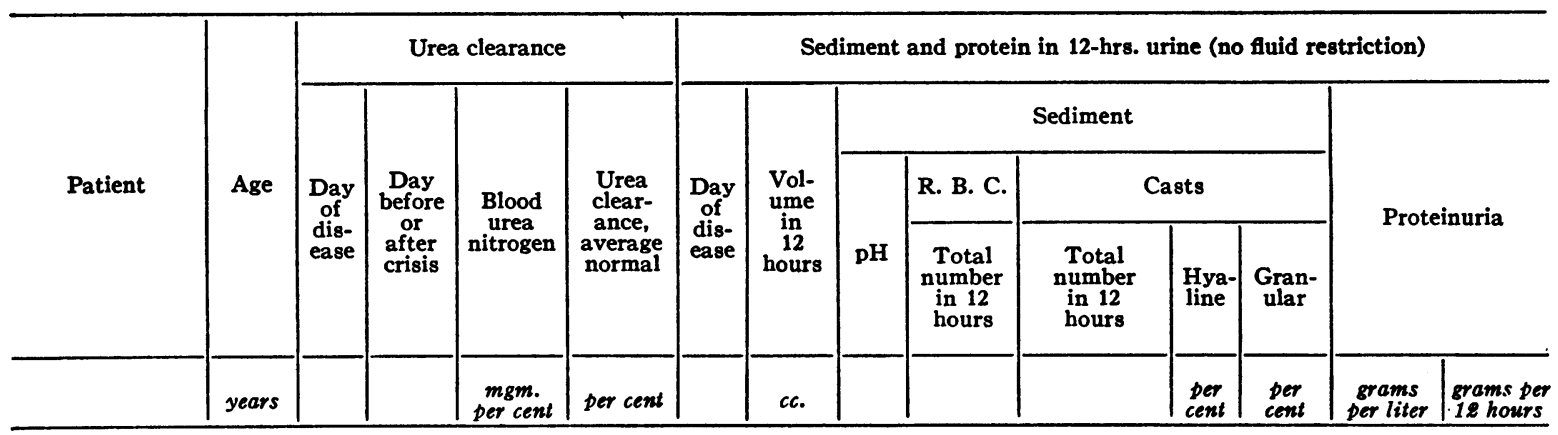

C. PATIENTS 40 TO 50 YeARS

\begin{tabular}{|c|c|c|c|c|c|c|c|c|c|c|c|c|c|c|}
\hline H. Y. ? & 40 & $\begin{array}{r}4 \\
9 \\
20 \\
43 \\
73 \\
109 \\
138\end{array}$ & $\begin{array}{c}0 \\
+5 \\
+16 \\
+39 \\
+69 \\
+105 \\
+124\end{array}$ & $\begin{array}{r}39.45 \\
20.25 \\
10.40 \\
8.29 \\
6.74 \\
7.92 \\
5.99\end{array}$ & $\begin{array}{l}10.3 \\
62.1 \\
82.1 \\
79.5 \\
98.6 \\
86.8 \\
82.3\end{array}$ & 7 & 720 & 5.0 & 200,000 & 500,000 & 34 & 66 & $<0.07$ & \\
\hline R. L. 9 & 42 & $\begin{array}{r}4 \\
8 \\
51 \\
105 \\
138\end{array}$ & $\begin{array}{c}0 \\
+4 \\
+47 \\
+101 \\
+134\end{array}$ & $\begin{array}{r}9.02 \\
14.63 \\
9.65 \\
13.30 \\
9.58\end{array}$ & $\begin{array}{r}87.0 \\
66.7 \\
63.0 \\
110.0 \\
78.6\end{array}$ & 9 & 312 & 5.0 & 60,000 & 16,000 & 100 & & $<0.07$ & \\
\hline M. S. $\%$ & 48 & $\begin{array}{r}3 \\
6 \\
13 \\
30 \\
64 \\
87 \\
107\end{array}$ & $\begin{array}{c}-3 \\
0 \\
+6 \\
+23 \\
+57 \\
+80 \\
+100\end{array}$ & $\begin{array}{r}12.48 \\
9.90 \\
19.35 \\
7.64 \\
12.27 \\
13.35 \\
10.26\end{array}$ & $\begin{array}{r}83.5 \\
131.1 \\
63.0 \\
121.2 \\
134.2 \\
147.8 \\
126.0\end{array}$ & $\begin{array}{r}3 \\
7 \\
12\end{array}$ & $\begin{array}{l}305 \\
360 \\
710\end{array}$ & $\begin{array}{l}4+ \\
8.0 \\
5.0\end{array}$ & $\begin{array}{c}0 \\
15,000 \\
0\end{array}$ & $\begin{array}{l}12,000 \\
35,000 \\
40,000\end{array}$ & $\begin{array}{r}100 \\
50 \\
100\end{array}$ & 50 & $\begin{array}{r}0.21 \\
<0.07 \\
<0.07\end{array}$ & 0.07 \\
\hline G. Z. 우 & 49 & $\begin{array}{r}3 \\
11 \\
16 \\
40 \\
51 \\
76 \\
91 \\
120\end{array}$ & $\begin{array}{l}-6 \\
+2 \\
+7 \\
+31 \\
+42 \\
+67 \\
+82 \\
+111\end{array}$ & $\begin{array}{r}21.28 \\
10.59 \\
13.54 \\
9.32 \\
14.51 \\
12.09 \\
16.99 \\
17.08\end{array}$ & $\begin{array}{r}49.0 \\
122.7 \\
59.7 \\
51.3 \\
51.6 \\
\\
70.5 \\
74.9\end{array}$ & $\begin{array}{r}3 \\
18\end{array}$ & $\begin{array}{l}210 \\
492\end{array}$ & $\begin{array}{l}4+ \\
4+\end{array}$ & $\begin{array}{l}\mathbf{0} \\
\mathbf{0}\end{array}$ & $\begin{array}{c}350,000 \\
0\end{array}$ & 10 & 90 & $\begin{array}{r}0.43 \\
<0.07\end{array}$ & 0.08 \\
\hline
\end{tabular}

D. PATIENTS 50 YEARS AND OVER

\begin{tabular}{|c|c|c|c|c|c|c|c|c|c|c|c|c|c|c|}
\hline A. I. ఠ্ & 52 & $\begin{array}{r}1 \\
6 \\
18 \\
58 \\
95\end{array}$ & $\begin{array}{r}0 \\
+5 \\
+17 \\
+57 \\
+94\end{array}$ & $\begin{array}{r}14.11 \\
11.53 \\
12.85 \\
9.84 \\
10.94\end{array}$ & $\begin{array}{r}101.2 \\
80.2 \\
92.3 \\
80.5 \\
95.2\end{array}$ & $\begin{array}{l}2 \\
7\end{array}$ & $\begin{array}{l}330 \\
660\end{array}$ & $\begin{array}{l}7.0 \\
5.0\end{array}$ & $\begin{array}{l}\mathbf{0} \\
\mathbf{0}\end{array}$ & $\begin{array}{l}\mathbf{0} \\
\mathbf{0}\end{array}$ & & & $\begin{array}{l}<0.07 \\
<0.07\end{array}$ & \\
\hline H. N. ? & 55 & $\begin{array}{r}2 \\
6 \\
12 \\
16 \\
26 \\
38 \\
77\end{array}$ & $\begin{array}{l}-3 \\
+1 \\
+7 \\
+11 \\
+21 \\
+33 \\
+72\end{array}$ & $\begin{array}{r}11.40 \\
13.15 \\
16.29 \\
19.90 \\
11.93 \\
11.04 \\
6.82\end{array}$ & $\begin{array}{r}115.6 \\
98.3 \\
72.7 \\
59.9 \\
109.9 \\
98.6 \\
80.0\end{array}$ & $\begin{array}{r}4 \\
12\end{array}$ & $\begin{array}{l}900 \\
500\end{array}$ & $\begin{array}{l}6.0 \\
4+\end{array}$ & $\begin{array}{l}50,000 \\
50,000\end{array}$ & $\begin{array}{r}1,400,000 \\
50,000\end{array}$ & $\begin{array}{r}25 \\
100\end{array}$ & 75 & $\begin{array}{r}0.86 \\
<0.07\end{array}$ & 0.78 \\
\hline M. C. $\sigma^{7}$ & 56 & $\begin{array}{r}1 \\
8 \\
18 \\
59 \\
152\end{array}$ & $\begin{array}{l}-6 \\
+2 \\
+12 \\
+53 \\
+146\end{array}$ & $\begin{array}{l}16.89 \\
24.48 \\
15.31 \\
18.47 \\
19.54\end{array}$ & $\begin{array}{l}75.9 \\
63.5 \\
98.2 \\
89.3 \\
87.5\end{array}$ & $\begin{array}{r}3 \\
19\end{array}$ & $\begin{array}{l}375 \\
650\end{array}$ & $\begin{array}{l}4+ \\
6.0\end{array}$ & $\begin{array}{c}25,000 \\
0\end{array}$ & $\begin{array}{l}45,000 \\
18,000\end{array}$ & $\begin{array}{r}75 \\
100\end{array}$ & 25 & $\begin{array}{r}0.14 \\
<0.07\end{array}$ & 0.05 \\
\hline
\end{tabular}


TABLE II-Continued

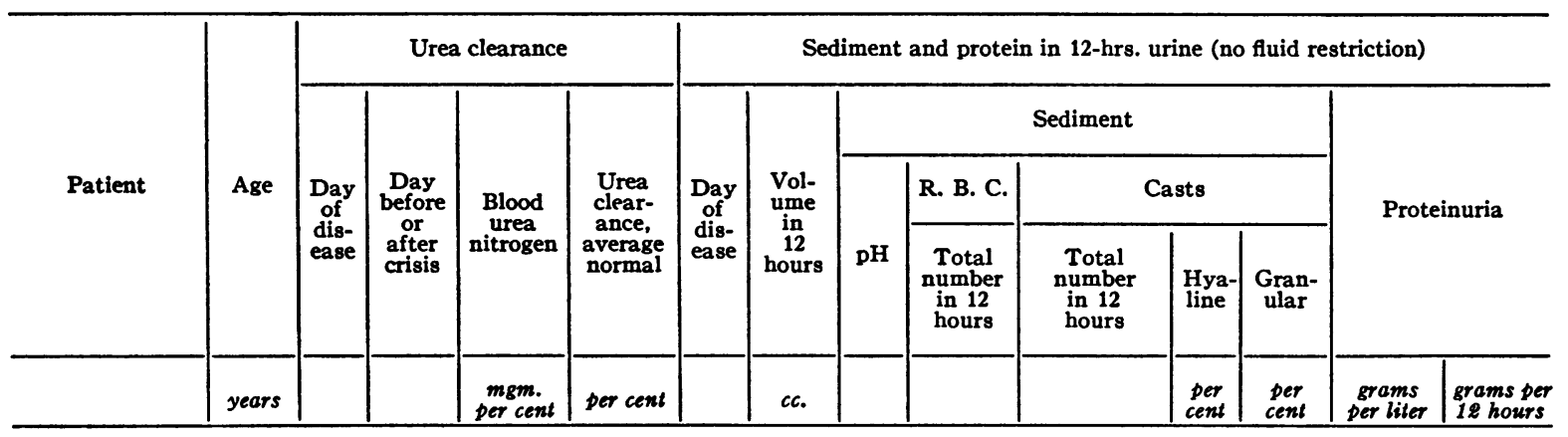

D. PATIENTS 50 Years AND OVER-Continned

\begin{tabular}{|c|c|c|c|c|c|c|c|c|c|c|c|c|c|}
\hline S. M. $\sigma^{T}$ & 56 & \begin{tabular}{|r|}
5 \\
10 \\
16 \\
31 \\
55 \\
113 \\
135
\end{tabular} & $\begin{array}{c}-4 \\
+1 \\
+7 \\
+22 \\
+46 \\
+104 \\
+126\end{array}$ & $\begin{array}{l}61.40 \\
17.08 \\
17.96 \\
10.95 \\
12.47 \\
12.14 \\
15.88\end{array}$ & $\begin{array}{r}24.3 \\
82.5 \\
69.0 \\
97.8 \\
73.5 \\
119.1 \\
68.2\end{array}$ & $\begin{array}{r}8 \\
13 \\
18\end{array}$ & $\begin{array}{l}633 \\
890 \\
590\end{array}$ & $\begin{array}{l}5.0 \\
7.0 \\
6.0\end{array}$ & $\left|\begin{array}{c}0 \\
0 \\
180,000\end{array}\right|$ & $\begin{array}{l}20,000 \\
30,000 \\
18,000\end{array}$ & $\begin{array}{l}100 \\
100 \\
100\end{array}$ & $\begin{array}{r}0.14 \\
<0.07 \\
<0.07\end{array}$ & 0.08 \\
\hline H. B. $\sigma^{7}$ & 65 & $\begin{array}{r}1 \\
5 \\
10 \\
52 \\
119\end{array}$ & $\begin{array}{l}-2 \\
+3 \\
+8 \\
+50 \\
+117\end{array}$ & $\begin{array}{l}11.28 \\
10.52 \\
13.72 \\
10.06 \\
11.72\end{array}$ & $\begin{array}{l}93.0 \\
74.8 \\
83.8 \\
67.3 \\
81.5\end{array}$ & $\begin{array}{l}6 \\
9\end{array}$ & $\begin{array}{l}\mathbf{5 7 5} \\
790\end{array}$ & $\begin{array}{l}8.0 \\
6.0\end{array}$ & $\begin{array}{c}16,000 \\
0\end{array}$ & $\begin{array}{c}32,000 \\
0\end{array}$ & 100 & $\begin{array}{l}<0.07 \\
<0.07\end{array}$ & \\
\hline
\end{tabular}

E. PATIENTS WHO DIED

\begin{tabular}{l|l|l|l|l|r|r|r|r|r|r|r|r|r|r}
\hline E. W. $\sigma^{7}$ & 45 & 3 & & 22.13 & 70.6 & 4 & 285 & $4+$ & 120,000 & $3,250,000$ & 0 & 100 & 4.82 & 1.37 \\
\hline L. G. $\sigma^{7}$ & 50 & 3 & & 30.5 & 50.1 & 4 & 480 & $4+$ & 0 & $2,000,000$ & 0 & 100 & 0.72 & \\
\hline
\end{tabular}

small samples of dilute urines must be borne in mind when interpreting these data. Casts, however, were uniformly increased in number with the hyaline type predominating. When the patients were extremely ill the proportion of granular casts increased. In the two patients who died, the differential cast count revealed 100 per cent granular casts with a large number of casts excreted in both instances.

Estimations of blood pressure. There was no significant alteration in the blood pressure accompanying the alteration in the urea clearance. In general, the blood pressure tended to be a little below normal during the febrile period of the disease, returning to the usual normal levels during convalescence.

Additional laboratory findings. Daily measurements of fluid intake and output were made on these patients and no deviations from the expected relations were noted. There was no relation between fluid intake and clearance, or be- tween fluid intake and the number of observed formed elements in the urine. Some of the patients were given sodium chloride in therapeutic doses. This had no demonstrable effect on the urea clearance in these patients.

\section{Relation of renal physiology to pneumococcus type and therapy}

There was no demonstrable difference between the effects of different pneumococcal types on renal function. Nor did the early and effective use of therapeutic sera have any demonstrable effect on the renal function during the acute illness and also during serum disease. No effect was visible when treated and untreated patients in the same age groups were compared. It is possible that statistical analysis of a larger number of cases might show some correlation between pneumococcus type and changes in renal function. If any connection was present in our cases, however, it was quite obscured by the more marked 
effects of age and increased lability of the renal function.

\section{DISCUSSION}

The outstanding revelation of these observations seems to be a decrease of renal resiliency with increasing age. The failure of older patients to develop a high urea clearance seems to be a part of their general loss of physiological elasticity. It must be emphasized here that normal children do not show urea clearances which, per square meter of body surface, exceed those of adults. It has been our experience, and also that of Cullen, Nelson and Holmes (14), that the variation in normal children from the mean, designated as " 100 per cent normal," follows the same pattern as in adults. However, a marked response to alteration of some physiological factors governing the urea clearance indicating a high degree of resiliency of renal function has been observed in young children with nephrosis (Farr (15)).

The effect of age on renal behavior in pneumonia may explain, in part, the differing conclusions of previous investigators on the effect of pneumonia on renal function.

Our observations indicate no significant renal damage caused by the hyperpyrexia or the toxemia of the disease. It seems likely that the albuminuria observed in this disease is more closely allied to the benign albuminuria noted after severe exercise or cold showers than to the serious albuminuria seen in Bright's disease.

The fact that the urea clearance did not return to normal promptly with the temperature makes it seem unlikely that the clearance elevation noted is related to increase in metabolism associated with fever.

Prompt response to serum therapy did not appear to hasten the return of the inflated clearance to normal.

That hyperpyrexia of itself does not increase the urea clearance was shown by Farr and Moen (16) who observed a decrease in the clearance initially when artificial pyrexia was induced. Some factor other than fever must cause the increase in urea clearance in pneumonia.

These studies shed no light on the mechanism by which Bright's disease may follow pneumonia, as none occurred in this group.
It seems possible that signs of renal irritation, such as red cell excretion and albuminuria, may be kept to a minimum by adequate administration of fluid.

Administration of salt to some of these patients had no appreciable effect upon the apparent renal function.

\section{CONCLUSIONS}

1. Renal physiological studies have been made upon 28 patients with lobar pneumonia.

2. The urea clearance during the disease shows a sharp difference between the older and younger patients. The patients under 40 years of age show a marked elevation of the urea clearance, persisting about one month, and independent of the type of pneumonia or therapy used. The older age group show little change in the urea clearance.

3. Observation of the urine sediment in all groups showed a strong tendency for all abnormal elements to disappear with adequate fluid balance.

4. No significant changes in blood pressure were associated with the clearance changes.

\section{BIBLIOGRAPHY}

1. Lewis, D. S., The clinical value of Ambard's coefficient of urea excretion. Arch. Int. Med., 1917, 19, 1.

2. McIntosh, J. F., and Reimann, H. A., Kidney function in pneumonia. J. Clin. Invest., 1926, 3, 123.

3. Goldring, W., Studies of the kidney in acute infection. III. Observations with the urine sediment count (Addis) and the urea clearance test in lobar pneumonia. J. Clin. Invest., 1931, 10, 355.

4. Rackemann, F. M., Longcope, W. T., and Peters, J. P., The excretion of chlorids and water and the renal function in serum disease. Arch. Int. Med., 1916, 18, 496.

5. Frothingham, C., Studies on renal function during and immediately following some of the acute infectious diseases. Arch. Int. Med., 1918, 22, 74.

6. Tileston, W., and Comfort, C. W., Jr., The total non-protein nitrogen and the urea of the blood in health and in disease as estimated by Folin's methods. Arch. Int. Med., 1914, 14, 620.

7. Schwartz, H., and McGill, C., Blood urea determinations in 211 cases. Arch. Int. Med., 1916, 17, 42.

8. Bookman, A., A study of renal function in patients convalescing from acute fevers. Arch. Int. Med., 1917, 20, 112.

9. Blackman, S. S., Jr., Pneumococcal lipoid nephrosis and the relation between nephrosis and nephritis. I. Clinical and anatomical studies. Bull. Johns Hopkins Hosp., 1934, 55, 1. 
10. Rake, G., Nephritis in pneumococcal infections. Guy's Hosp. Rep., 1933, 83, 430.

11. Seegal, D., Acute glomerulonephritis following pneumococcic lobar pneumonia. Arch. Int. Med., 1935, 56, 912.

12. Winkenwerder, W. L., McLeod, N., and Baker, M., Infection and hemorrhagic nephritis. Arch. Int. Med., 1935, 56, 297.

13. Shevky, M. C., and Stafford, D. D., A clinical method for the estimation of protein in urine and other body fluids. Arch. Int. Med., 1923, 32, 222.
14. Cullen, G. E., Nelson, W. E., and Holmes, F. E., Studies of kidney function in children. I. Urea clearance values: (1) no evidence of kidney disease, (2) after acute hematuric nephritis following an acute infection, (3) in the acute stage of hematuric nephritis. J. Clin. Invest., 1935, 14, 563.

15. Farr, L. E., The effect of dietary protein on the urea clearance of children with nephrosis. J. Clin. Invest., 1936, 15, 703.

16. Farr, L. E., and Moen, J. K. (In preparation.) 